\title{
Utilizando uma Rede Neural Artificial ARTMAP-Fuzzy para Realizar o Diagnóstico Clínico de Amostras de Câncer de Mama
}

\author{
$\underline{\text { James C. Silva }}^{\dagger}$ Fernando P. A. Lima $^{\dagger}$ Mara L. M. Lopes ${ }^{*}$ Carlos R. Minussi $^{\dagger}$ \\ ${ }^{\dagger}$ Departamento de Engenharia Elétrica, Faculdade de Engenharia de Ilha Solteira (FEIS) \\ *Departamento de Matemática, Faculdade de Engenharia de Ilha Solteira (FEIS) \\ Universidade Estadual Paulista "Júlio de Mesquita Filho" (UNESP), Ilha Solteira, SP, BRASIL \\ E-mails: jamesclauton@gmail.com, engfernandoparra@gmail.com, \\ mara@mat.feis.unesp.br, minussi@dee.feis.unesp.br
}

Palavras-chave: Diagnóstico, Câncer de Mama, Redes Neurais Artificiais, ARTMAP-Fuzzy, WBCD.

Resumo: Neste artigo apresenta-se um método para o diagnóstico de amostras de câncer de mama utilizando uma rede neural artificial ARTMAP-Fuzzy, a qual é responsável por discriminar as amostras, obtendo uma classificação em casos benignos ou malignos. A principal aplicação desta metodologia é auxiliar os profissionais no processo de diagnóstico de câncer de mama, visando rapidez na tomada de decisão, confiabilidade e a assistência necessária para salvar vidas. Para avaliar este método, utilizou-se a base de dados Wisconsin Breast Cancer Diagnosis (WBCD), que é composta por dados reais de câncer de mama. Os resultados obtidos pelo método são considerados satisfatórios comparados com a literatura.

\section{Introdução}

O câncer é uma doença crônica que atinge milhões de pessoas em todo o mundo. Segundo um levantamento realizado pelo Instituto Nacional de Câncer (INCA) no ano de 2012, evidenciouse que o câncer de mama é o segundo mais frequente no mundo e o mais comum entre as mulheres, respondendo por $22 \%$ dos casos novos a cada ano. No ano de 2012 também foram diagnosticados 52.680 novos casos de câncer de mama no Brasil [3]. O câncer de mama é o que mais leva os pacientes a óbito, aproximadamente 39\% das mulheres que lutam contra o câncer de mama, morrem [9].

O diagnóstico correto em um estado prematuro do câncer de mama pode auxiliar na tomada de decisões, no planejamento de ações e, evidentemente, na eficiência do tratamento. No entanto, diagnosticar corretamente o câncer é um processo complexo e muito difícil, em consequência das diversas variáveis envolvidas. Para um diagnóstico preciso, exige-se muita experiência e, principalmente, que a classificação do estadiamento clínico do tumor (estágio do câncer) esteja correta.

Os tradicionais sistemas de classificação de patologias utilizados pelos médicos são complexos, e normalmente de difícil utilização, oferecendo muitas limitações [6]. Desta forma, torna-se necessário o desenvolvimento de sistemas integrados que trabalhem com técnicas de processamento e análise de dados, e que, combinados com a experiência dos profissionais, proporcione a assistência necessária para realizar o diagnóstico preciso da doença.

Neste sentido, a aplicação de métodos baseados em computação inteligente (CI) torna-se uma possível solução para o problema de diagnóstico. Os métodos inteligentes são capazes de extrair informações e conhecimento de problemas complexos, e são de fácil aplicação. $\mathrm{Na}$ sequência apresentam-se os trabalhos mais relevantes sobre diagnóstico de câncer de mama disponível na literatura.

No artigo [10], os autores apresentam um método para diagnóstico de amostras de câncer utilizando a lógica fuzzy e o algoritmo genético. Em [11] foi usada uma rede ANFIS para realizar o diagnóstico de câncer de mama. No artigo [8] foi utilizado um sistema híbrido com uma rede neural e um sistema especialista fuzzy. Em [4] os autores apresentam um método 
baseado no algoritmo de seleção negativa para o diagnóstico de câncer de mama. Os autores citados fizeram uma série de testes e obtiveram bons resultados.

Neste artigo, apresenta-se um método para diagnóstico de câncer de mama utilizando uma rede neural artificial baseada na teoria da ressonância adaptativa, denominada rede ARTMAPFuzzy. Optou-se por empregar tal arquitetura pela sua estabilidade, plasticidade e eficiência em relação as demais redes, pois além de obter um treinamento rápido em relação a maioria das outras redes neurais. $\mathrm{O}$ processo de treinamento é realizado instantaneamente. Esta arquitetura permite a inclusão do módulo de treinamento continuado, ou seja, podem-se inserir novos padrões sem a necessidade de reiniciar o treinamento. Desta forma, o principal objetivo é aplicar a rede ARTMAP-Fuzzy nas amostras cancerígenas da base de dados Wisconsin Breast Cancer Diagnosis[12] [13], que contém dados reais sobre o câncer de mama.

\section{Base de Dados Wisconsin Breast Cancer Diagnosis (WBCD)}

A base de dados WBCD foi criada pelo Dr. William H. Wolberg em seu ofício, entre os anos de 1989 e 1991 no hospital escola da Universidade de Wisconsin Madison. A base de dados contém 9 instâncias representando as características do tumor e, evidentemente, a classificação para estas instâncias, totalizando 10 variáveis [12].

As características armazenadas na base de dados são as seguintes [5]:

1. espessura da massa celular (CT);

2. uniformidade do tamanho da célula (CS);

3. uniformidade do formato da célula $(\mathrm{CH})$;

4. adesão marginal (AD);

5. tamanho de uma célula epitelial (EP);
6. núcleo vazio $(\mathrm{BN})$;

7. cromatina branda $(\mathrm{CO})$;

8. nucléolo normal $(\mathrm{NN})$;

9. mitose (MM);

10. classificação ("benigno" ou "maligno").

Esta base de dados possui 699 amostras, sendo que $65 \%$ representam tumores benignos e $35 \%$ representam tumores malignos [13].

\section{ARTMAP-Fuzzy}

A rede neural ARTMAP-Fuzzy corresponde a um sistema de aprendizado supervisionado composto por um par de módulos da teoria da ressonância adaptativa, ARTa-Fuzzy e ARTbFuzzy, conectados pelo módulo de memória associativa inter-ART. Esta rede incorpora a teoria dos conjuntos fuzzy, operador fuzzy AND $\left({ }^{\wedge}\right)$, capacitando o aprendizado do sistema neural em resposta a padrões de entrada binários e analógicos. A Figura 1 ilustra a arquitetura da rede neural ARTMAP-Fuzzy [2].

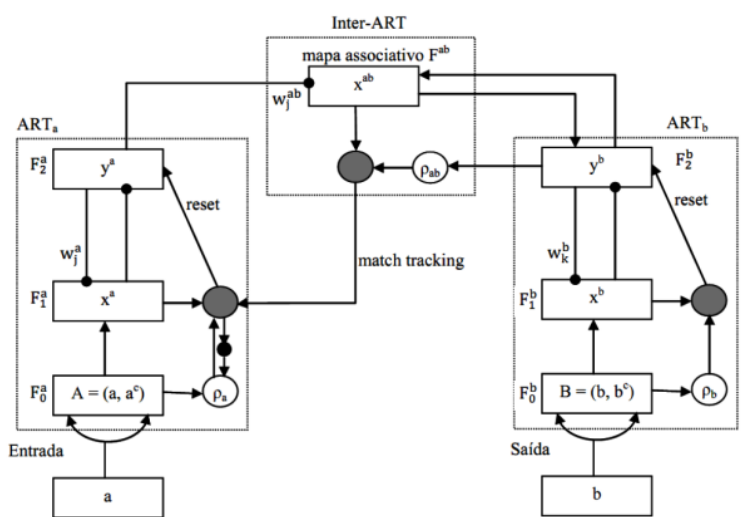

Figura 1 - Arquitetura da rede neural ARTMAP-Fuzzy [2].

Um mecanismo interno denominado match tracking é responsável pelo processo autorregulador da rede, no qual é maximizada a generalização e minimizado o erro. Sempre que a rede realiza um prognóstico errado, através de uma conexão associativa instruída, o parâmetro 
de vigilância do módulo ARTa-Fuzzy é incrementado em uma quantidade mínima necessária para corrigir o erro no módulo ARTb-Fuzzy.

Existem três parâmetros fundamentais no desenvolvimento da rede neural ARTMAPFuzzy [2], são eles:

- Parâmetro de escolha $\alpha(\alpha>0)$

: atua na seleção de categorias;

- Taxa de treinamento $\beta\left(\beta \in\left[\begin{array}{ll}0 & 1\end{array}\right]\right) \quad$ : controla a velocidade da adaptação da rede;

- Parâmetro de vigilância $\rho\left(\rho \in\left[\begin{array}{ll}0 & 1\end{array}\right]\right)$ : controla a ressonância da rede. Trata-se do parâmetro responsável pelo número de categorias criadas.

Se $\rho$ possui um valor grande, a rede torna-se mais seletiva reduzindo a capacidade de generalização. Caso $\rho$ tenha um valor pequeno, reduz-se o número de categorias criadas, aumentando a capacidade de generalização.

\section{Metodologia Proposta}

O sistema de diagnóstico de câncer de mama é baseado em uma rede neural artificial ARTMAP-Fuzzy. Este sistema é o responsável por realizar a discriminação das amostras de câncer, entre benigno e maligno.

O método proposto é executado em duas fases: treinamento e o diagnóstico dos dados. $\mathrm{Na}$ sequência apresenta-se o conjunto de testes e o esquema de funcionamento da rede ARTMAPFuzzy no diagnóstico de câncer de mama.

\subsection{Conjunto de testes}

A base WBCD possui 10 atributos, no entanto, para este artigo optou-se por utilizar 5 atributos. Para escolher os atributos, utilizou-se o cálculo do desvio padrão das amostras. Foram escolhidos os 5 atributos que apresentaram os índices mais baixos de desvio padrão, isto é, os dados mais homogêneos. Esta estratégia foi adotada visando maior confiabilidade.

O desvio padrão foi calculado utilizando a Equação (1):

$$
S=\sqrt{\frac{1}{n-1} \sum_{i=1}^{n}\left(z_{i}-\bar{z}\right)^{2}}
$$

em que $n$ é o número total de amostras, z são as amostras envolvidas e $\bar{z}$ é a média amostral.

Os atributos selecionados pela análise de menor desvio são: a espessura da massa celular (CT), a uniformidade do tamanho da célula (CS), a uniformidade do formato da célula $(\mathrm{CH})$, o núcleo vazio $(\mathrm{BN})$ e o nucléolo normal $(\mathrm{NN})$. Durante a separação destas variáveis observou-se que alguns dados não são aproveitáveis, ou seja, o desvio padrão foi muito próximo de zero.

Tabela 1 - Especificação da base de dados WBCD.

\begin{tabular}{lc}
\hline \multicolumn{2}{c}{ Base de dados WBCD } \\
\hline Tipo & Classificação \\
\hline Número de dados & 699 \\
\hline $\begin{array}{l}\text { Número de dados } \\
\text { aproveitáveis }\end{array}$ & 682 \\
\hline Dados da classe "benigno" & 444 \\
\hline Dados da classe "maligno" & 238 \\
\hline Número de atributos & 5 \\
\hline
\end{tabular}

Na Tabela 1 ilustra-se a especificação da base de dados WBCD usada no treinamento e diagnóstico da rede neural ARTMAP-Fuzzy.

\subsection{Sistema de Diagnóstico}

O sistema de diagnóstico de câncer de mama proposto neste artigo é baseado na rede ARTMAP-Fuzzy. O esquema de funcionamento deste sistema é ilustrado no diagrama de blocos apresentado na Figura 2. 


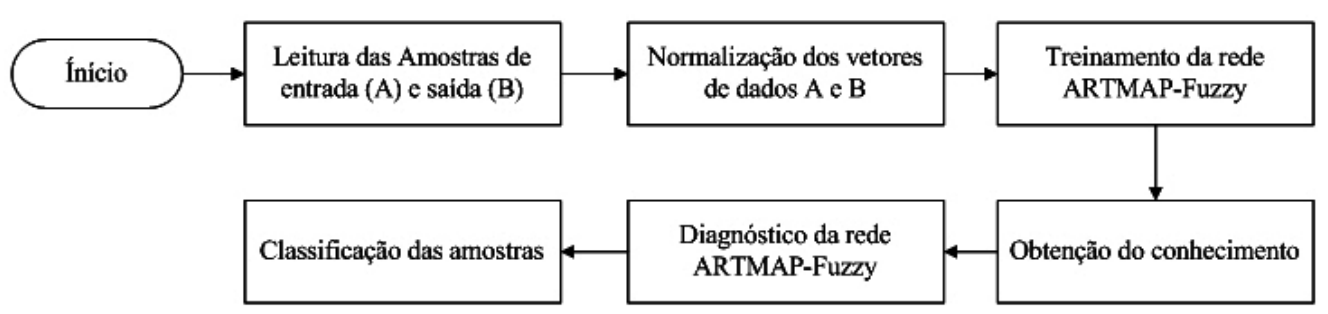

Figura 2 - Esquema de funcionamento do sistema de diagnóstico.

O módulo de leitura das amostras é responsável por fazer a aquisição dos dados WBCD. O conjunto de entrada de dados da rede neural ARTMAP-Fuzzy é composto pelos atributos da amostra de câncer de mama da base de dados WBCD (espessura da massa celular, uniformidade do tamanho da célula, uniformidade do formato da célula, o núcleo vazio e nucléolo normal $(\mathrm{NN})$ ). O conjunto de saída é formado pela classificação do conjunto de entrada (amostras cancerígenas) em benigno ou maligno.

O módulo de treinamento da rede neural ARTMAP-Fuzzy é realizado de forma off-line e serve para ajustar os pesos e criar as categorias (conhecimento) que serão utilizadas no diagnóstico dos dados. Neste módulo, a rede é treinada considerando-se um conjunto de dados e será gerada uma quantidade de categorias, no máximo, igual ao número de amostras de entrada.

Desta forma a rede ARTMAP-FUZZY utiliza o parâmetro $\alpha$ para escolha da categoria, sendo assim responsável por determinar que a rede busque a menor categoria criada para esse padrão, já o $\beta$ é importante para determinar a velocidade do treinamento, fazendo assim que a rede utilize mais tempo treinando um determinado padrão a ser utilizado na categoria. E o parâmetro $\rho$ será utilizado para a criação das categorias, ou seja, à partir dele é que sé determinado a quantidade de categorias e se um padrão é semelhante o bastante para que seja incluso em uma categoria já existe.

O módulo de diagnóstico é realizado de forma on-line. Neste fase, um novo conjunto de amostras, diferente do conjunto de dados utilizados na fase de treinamento, deve ser apresentado à rede. Cada amostra será analisada individualmente. Todas as amostras são comparadas com o conhecimento (categorias criadas na fase de treinamento) extraído pela rede neural ARTMAP-Fuzzy. Assim, quando houver um casamento entre os padrões classifica-se em categoria a amostra apresentada a rede ARTMAP-Fuzzy. Obtida a categoria correspondente a um determinado padrão é, portanto, identificado o diagnóstico, que é a classificação da amostra cancerígena como sendo benigna ou maligna.

\section{Resultados}

Os testes (simulações) foram realizados utilizando um PC Intel Core 2 Duo $1.9 \mathrm{GHz}, 2 \mathrm{~GB}$ de Memória RAM, e sistema operacional Windows 7 Ultimate 32 bits. O algoritmo foi desenvolvido em MATLABß [7].

Os parâmetros da rede neural ARTMAP-Fuzzy utilizados no processo de treinamento e diagnóstico foram: $\alpha=0,1 ; \beta=1$ (treinamento rápido); $\rho_{\mathrm{a}}=0,8 ; \rho_{\mathrm{b}}=1 \mathrm{e} \rho_{\mathrm{ab}}=1$.

$\mathrm{Na}$ fase de treinamento foi utilizado um conjunto de dados com 341 amostras, sendo 222 amostras em condições normais (benigno) e 119 amostras cancerígenas (maligno). Este conjunto de dados corresponde a 50\% dos dados disponíveis. Para avaliar a rede neural ARTMAP-Fuzzy na fase de diagnóstico foi utilizado um conjunto de dados com 341 amostras, sendo 222 amostras benignas e 119 amostras malignas. Estes dados utilizados nos testes representam 50\% dos dados disponíveis. A base de dados simulada conta com 682 amostras, resumidas em 2 padrões diferentes, benigno e maligno.

A Equação (2) fornece o cálculo para a análise do percentual de acerto obtido pelo diagnóstico da rede neural ARTMAP-Fuzzy. 


$$
\text { acertos }=\frac{t * 100}{t+f}
$$

em que $t$ representa o total de acertos e $f$ o total de erros.

Na Tabela 2 apresentam-se os resultados obtidos pelo sistema de diagnóstico de câncer de mama aplicados à base de dados WBCD.

Tabela 2 - Resultados obtidos pelo método.

\begin{tabular}{c|c|c}
\cline { 2 - 3 } & $\begin{array}{c}\text { Fase de } \\
\text { Treinamento }\end{array}$ & Fase de Diagnóstico \\
\hline Amostras Utilizadas & 341 & 341 \\
\hline Classificações Corretas & 341 & 322 \\
\hline Classificações Erradas & 0 & 19 \\
\hline Acerto (\%) & 100,00 & 94,43 \\
\hline Tempo (s) & 4,6 & 1,07 \\
\hline
\end{tabular}

Para obtenção destes resultados, o sistema foi submetido a uma fase de testes e ajustes de parâmetros. Os resultados apresentados foram obtidos com a melhor configuração da rede neural ARTMAP-Fuzzy. Foram analisados dois parâmetros, a sensibilidade (S) e a especificidade (E) do método que são dadas pelas seguintes equações:

$$
\begin{aligned}
& S=\frac{V P}{V P+F P} \\
& E=\frac{V N}{F N+V N}
\end{aligned}
$$

sendo:
$V P$ : verdadeiro
$V N$ : verdadeiro
$F P$ : falso
FN : falso
positivo;
negativo;
positivo;
negativo.

Foram realizados 25 testes com variações nos valores de cada parâmetro, obtendo, assim, sua melhor configuração. Ao variar o parâmetro $\alpha$ em 0,2 entre 0,1 e $1, \rho_{\mathrm{a}}, \rho_{\mathrm{b}}, \rho_{\mathrm{ab}}$ em 0,05 entre 0,8 e 1 não se obteve mudança nos valores de sensibilidade e especificidade. Porém, quando o parâmetro $\beta$ é variado ocorre uma diferença muito grande no valor de especificidade, ficando entre $35 \%$ a $70 \%$. Como consequência desta análise tem-se que algumas pessoas sem a doença estariam sendo indicadas para a realização de exames mais específicos.

Para $\beta$ com valor igual a 1, a rede neural artificial ARTMAP-Fuzzy obtêm seu melhor resultado, atingindo um valor de especificidade de $91,44 \%$, neste caso, somente $8,56 \%$ dos indivíduos sem a doença estariam sendo recomendados para um exame mais detalhado. Para os testes realizados, o valor de sensibilidade obtido foi de $100 \%$, indicando que todos os indivíduos que possuem a doença seriam submetidos a exames mais detalhados, e consequentemente, ao tratamento.

Neste sentido, a rede neural ARTMAP-Fuzzy apresentou um bom desempenho, ou seja, nenhum paciente ficou sem assistência medica, caso fosse indicado, por este método, a suspeita de malignidade.

\section{Conclusão}

Neste artigo foi apresentado um método de diagnóstico de câncer de mama utilizando uma rede neural artificial ARTMAP-Fuzzy. O método proposto apresentou bons resultados obtendo um índice de acerto superior a $94 \%$ para a melhor configuração da rede. O diagnóstico obtido através da rede neural ARTMAP-Fuzzy não deixou de detectar nenhum caso de câncer, isto é muito importante, do ponto de vista de salvar vidas. A rede ARTMAP-Fuzzy apresentou um processamento rápido, com tempo inferior a 100 milésimos de segundo, o que credencia o algoritmo a ser empregado em tempo real, na tomada de decisões. Desta forma, conclui-se que 
a rede neural artificial ARTMAP-Fuzzy usada neste artigo é bastante eficiente, confiável, robusta e precisa, quando aplicada no diagnóstico de amostras câncer de mama. Vale ressaltar que o método apresentado neste trabalho é puro, não utilizando outras técnicas em conjunto, diferentemente da maioria dos trabalhos disponíveis na literatura.

Como trabalhos futuros, pretende-se analisar outros tipos de doenças, empregando a mesma metodologia, baseada na rede neural ARTMAP-Fuzzy; pretende-se também aprimorar esta técnica, compondo uma metodologia hibrida, utilizando sistemas imunológicos artificiais e lógica fuzzy.

\section{Agradecimentos}

Os autores agradecem a CAPES e a FAPESP (Proc. N 2011/06394-5) pelo apoio financeiro de pesquisa e ao Centro de Aprendizado de Máquina e Sistemas Inteligentes da Universidade da Califórnia, (UCI - Machine Learning Repository) por disponibilizar a base de dados WBCD.

\section{Referências}

[1] F. Camastra. Kernel Methods For Clustering. In WIRN/NAIS, volume 3931 of Lecture Notes in Computer Science, pp. 1-9 (2006).

[2] G. A. Carpenter, S. Grossberg, N. Markuzon, J. H. Reynolds, D. B. Rosen, "Fuzzy ARTMAP: A Neural Network Architecture for Incremental Supervised Learning of Analog Multidimensional Maps", IEEE Trans. on Neural Networks, Vol. 3, No. 5, pp. 698713 (Sept. 1992).

[3] INCA - Instituto Nacional do Câncer (Brasil), disponível em: www.inca.gov.br.

[4] F. P. A. Lima; M. L. M. Lopes; A. D. P. Lotufo e C. R. Minussi. Diagnóstico Clínico de Amostras de Câncer de Mama utilizando Sistemas Imunológicos Artificiais com Seleção Negativa, SBAI - Simpósio Brasileiro de Automação Inteligente, Fortaleza, pp. 1-6 (2013).

[5] O. L. Mangasarian; R. Setiono and W. H. Wolberg. Pattern recognition via linear programming: Theory and application to medical diagnosis, in: "Large-scale numerical optimization", Thomas F. Coleman e Yuying Li, editores, Publicações SIAM, Philadelphia 1990, pp. 22-30 (1990).

[6] K. Manikantan; S. I. Sayed; K. N. Syrigos; P. Rhys-Evans; C. M. Nutting; K. J. Harrington and R. Kazi. Challenges For The Future Modifications of The TNM: Staging System For Head And Neck Cancer. Cancer Treatment Reviews, pp. 35-44 (2009).

[7] Matlab (2011). 7.8 Version, Mathworks Company.

[8] P. Meesad and G. G. Yen. Combined Numerical and Linguistic Knowledge Representation and Its Application to Medical Diagnosis, IEEE Transactions on Systems, Man, and Cybernetics- Part A: Systems And Humans, Vol. 33, No. 2, pp206-222 (March 2003).

[9] OMS - Organização Mundial da Saúde, Disponível em: http://www.who.int/en/.

[10] C. A. Peña-Reyes and M. Sipper. Designing Breast Cancer Diagnostic System via Hybrid Fuzzy-Genetic Methodology, 1999 IEEE International Fuzzy Systems Conference Proceeding, Seoul-Korea, pp. I135-I139.(August 1999).

[11] J. Wang and C. S. George Lee. Self-Adaptive Neuro-Fuzzy Inference Systems for Classification Applications, IEEE Transactions on Fuzzy Systems, pp. 790-802 (2002).

[12] WBCD, (2012). Wisconsin Breast Cancer Data - UCI Machine Learning Repository, disponível em: www.arquives.ics.uci.edu/ml/

[13] W. H. Wolberg and O. L. Mangasarian. Multisurface Method of Pattern Separation For Medical Diagnosis Applied to Breast Cytology, Proceedings of National Academy of Sciences (USA), Vol. 87, pp. 9.193-9.196 (1990). 